\title{
A Literature Survey on Intrusion Detection System in Manets using Machine Learning Techniques
}

\author{
Tarik Fouad Himdi, PhD \\ King Abdulaziz University, Jeddah, \\ Kingdom of Saudi Arabia
}

\begin{abstract}
Since, a decade of time Mobile Ad hoc Networks (MANETs) have come with wireless networking technology. Due to its dynamic in nature of MANETs, these are vulnerable to various attacks in all the OSI layers but research shows that in Network layer the attacks are effectively done by intruders. In this survey many of the attacks at Network layer are identified in MANETs by most of the researchers which is outlined in this paper. Mostly, AODV routing protocols and other protocols are used for transferring packets in the direction of the destination. These transferred packets data is then deposited within the log files, to surveillance these routing of packets from these
\end{abstract}

Log files, the techniques used in MANETs are Data mining, Support Vector Machines (SVM), Genetic algorithms (GA) and other Machine learning approaches. Further, the methodologies and techniques proposed for detecting and predicting these attacks from various kinds of intrusions within the MANETS is discussed.

\section{Keywords}

Mobile Adhoc Networks (MANETS), Intrusion Detection System (IDS), Support Vector Machines (SVM), Genetic algorithms (GA), Ad-hoc On-Demand Distance Vector (AODV).

\section{INTRODUCTION}

Today globally wireless set of connections are enormously spreading and used by many people due to its immense necessity and usage of mobile devices. MANETs must be the approaches which will be sound and safe for the broadcasting and communication of information that has become a relatively complex which is of very important concern[2][3]. Manets are a cluster of multiple mobile nodes that make use of wireless interface from provisional networks. The significance of MANET is that it does not have any fixed infrastructure due to which there are more occurrences of intrusions in the Network and MANETS do not have any centralized control.

Intrusion detection the approach used to detect malicious activity through pattern recognition in enormous information set comprising the technique of Artificial intelligence and Machine Learning [3]. Intrusion detection is used for detecting any unauthorized access done for personal computer or any Thinking machine. Due to this, providing Security is becoming critical task with the growing of internet applications that make use of MANETs. The existing security can be enhanced and improved by using appropriate Intrusion Detection technique at specific network layer in which very little research is done. The patterns of End-user activities are examined and Intrusions are identified using SVM and Decision Trees (DT) [26].

\section{PROBLEMS OF MOBILE ADHOC NETWORKS IN WIRELESS NETWORK ENVIRONMENTS}

The Wireless Networking Technology with the developments of the MANETs are continuously updating like a key towards the next generation advancements. Many of the MANETs will be susceptible to the different attacks at most of the levels, especially in case of the network layer; during its construction of a large amount of MANET routing protocols take for granted that there is no malevolent trespasser point in the set of connections. But, Intrusion detection system has different hits in Mobile Ad hoc Networks as mentioned below.

- Particular based section

- Sign based detection

- $\quad$ Spoofing

\section{LITERATURE REVIEW}

In the MANET the idea of Prevention is not enough from the security point, therefore Intrusion Detection system is the one more another concept of facilitating the safety measures in the system, Intrusions Detection System is operated to recognize the egotistic and also malicious node of internet network. An IDS is a proficient as well as useful method used in MANETS for the discovery of malicious activity. An ID is one of the software application which is used to predict malicious nodes from network. Thus, to overcome these IDS are being installed on these nodes to avoid them in the MANET due its centralized behavior [1] [2]. The main categories of MANETS as discussed in [3].

- $\quad$ Signature based detection.

- Requirement based detection.

- Abnormality based detection.

Similarly, the IDS has representative type of attacks in the context of MANETS as discussed in [4] [5].

- Spoof

- Denial of Service attack

- Black hole attack

It is evaluated in this paper that, Intrusion Detection techniques are based on Intrusion Datasets. The dataset used by the author is Aegean Wi-Fi Intrusion detection Dataset. This dataset is tested by Machine Learning Techniques from which Feature based Reduction techniques is used. The Feature reduction technique gave a good Gain; similarly the statistics of Chi-Squared is applied for the evaluation of the dataset presentation along with attribute reduction [6].

Outcomes obtained are shown using the attribute reduction that may result in the better when compared to the accuracy it has resulted from 110 to 41 is 2.4 percent [6]. 
Categorization of Intrusion detection strategies are categorized as misuse-based detection, anomaly based detection also the amalgamation of two [7]. The mechanism of intrusion discovery has been misused therefore the mechanism compares network packet movement along with the known malevolent threat patterns. This technique gives accuracy of higher grade, but it has easy implementation, whereas it cannot detect unknown intrusions [8]. Anomaly based detection is capable of identifying unknown attacks but it has accuracy in lower state. The Continuous evaluation of Dataset is done to improve the Performance.

The methodology of Genetic Algorithm to select optimum protocol with the context based on network is proposed [9]. The comprehensive performance of three protocols is used such as DSR, OLSR and AODV. To optimize the performance the WPAN is linked with fuzzy logic techniques, Neuro fuzzy technique is used to improve the performance with each routing protocol. Similarly, in another study Genetic Algorithm is used to improve the Performance [9].

Another author has discussed in his paper about various detection techniques and the most effective is the Anomaly based Detection in which the author has concentrated over Machine learning Technique, which identifies the attack traffic in online, by rewriting the Intrusion detection system regulations upon a fly-air experimentations also carried out by making use of Dockemu emulation device along with the Linux Containers, IPV6 and OLSR as the Routing Protocol. The author has proposed a way towards the discovery engine for the Intrusion Detection System, wherein, it identifies the hit online mode by making use of Machine Learning technique. These techniques are based on Support Vector Machine and have been tested with the detailed research by making use of the Dockemu emulation application along with the Linux Containers, OLSR also IPV6 at the same time the Routing Protocol with due consideration of a realistic world research. This suggestion is victorious in the designing of the rule patterns for the classification of the Denial of service attack with exactness. Finally this method of approach has given a Support Vector Machine good improved accuracy and Performance [10]. The Manets in a general medium, altering topology, short of the infrastructure along with the restricted power supply they are also susceptible depending upon the elementary properties. In this Paper the author has discussed about detecting a malevolent node in MANET's by Secure Intrusion Detection System which uses Dynamic Source Routing Protocol, DSRP can be used to avoid the Forged Acknowledgement [11]. In one of the proposed method Dynamic Source Routing procedure is used which is DSRP. The same Dynamic Source Routing protocol will be utilized for wired set of groups also with the wireless networks randomly. Dynamic Source Routing procedures have been classified into the two processes which is as shown below [12].

\section{- Route discovery \\ - Way or path maintenance}

This is a technique in which the initial point required to transfer the packets to the final point this way discovery will be only used when there is a need for the initial node to transfer the packer to the final point. If any kind of failure is found in the link then it will send message to the initial node saying that there is an "ROUTE ERROR".

In case if the differentiation is done between the different packets of the data which are being used in the proposed system that includes 2 bit packet header in DSRP. There are few fixed bits in the Dynamic Source Routing Protocol. Below table A shows the different packets of different types along with the flags.

Table 1 Different Packet category and the flags

\begin{tabular}{|c|c|}
\hline Packet Category & Packet Flag \\
\hline Common Information & 00 \\
\hline Acknowledgement & 01 \\
\hline @CA & 10 \\
\hline DMNC & 11 \\
\hline
\end{tabular}

There are other add-On techniques used in Intrusion Detection System namely watchdog, 2-ACK and A-ACK but there exists minor problems in the schemes. To overcome another technique is proposed for the above 3 difficulties of the watchdog which has very few broadcast energy, recipient impact also wrong misbehaving node. During this, Dynamic Source Routing Rules are utilized and it uses the Cryptographic algorithm namely Rivert Shamir Adleman RSA to avoid the forged acknowledgement. In one of such study shows that by stringent checking of co-operative statistical anomaly recognition models can protect through the attacks on adhoc routing protocol and wireless MAC protocol as well as on wireless application services [13].

The system is integrated with cross layer defense system. The Intrusion detection takes the Audit data and performs the reasoning of the data and tries to identify whether the method is under hit. Intrusion detection is capable of different type either Network dependent or the Host dependent [14]. Network based IDS surveillance is the first step for network through which packets are then transferred into network hardware edge. Host dependent basically rely on operating system inventory data in order to monitor the data and also to analyze it [23].

\section{ATTACKS IN MANETS}

There are a variety of attacks on the network layer or interference which are very famous and are meant for Mobile Ad-hoc Networks. The categorization of the main network layer attacks and bring in few typical attacks are explored in this part [15]. Network layer hit in the Mob Adhoc Networks are classified into 2 attacks, which are named as active and passive attacks.

\subsection{Passive Attacks}

These are the attacks, in which the invader will never change the process of the direction-finding rules and will follow to look for some precious information via the traffic examination technique [15].

\subsection{Active Attacks}

The situation in the active attacks are like, invasive behavior will be mostly shot by the intruders. For example formulating, updating, counterfeiting, constructing, embedding, skipping data or routing packets that resulted in different types of errors inside the network. Few attacks are due to the only one attempt of an intruder [15].

\subsection{Eavesdropping}

As due to the wireless behavior in MANETs, transmitted message which is put up via the point and every other device will receive it assembled from the transceiver which is 
surrounded around the radio transmission series, also if none of such technique of coding is utilized that time only the attacker will receive the helpful information. The receiver as well as the sender is unaware of each other of falling prey to this attack [16].

\subsection{Black Hole Attack}

Intruders make use of weaknesses that exists in the direction of the discovery methods in on-demand routing protocols, for example, Dynamic Source Routing and AODV, where these specific node necessitates a designate path towards the final point. Then, this particular point will send a Route Request (R-REQ) and then this intruder interrupts to divert it to other node path as if it is the new route. Thus, through this repetition process for the route requests obtained from the other nodes, the intruder will win by participating as one of the part of routes in the network. Hence, it will then become the mediator between the two nodes as the intermediate point and starts dropping of packets instead of pushing to other nodes, creating a black hole attack (BH) [17] [19].

\subsection{Grey Hole Attack}

Black hole attacks extraordinary case is Grey Hole Attack, in this primarily the intruder identifies all existing possible paths in the network, and proceed to participate as one of the important part of these paths in the connections which is similar in case of the BH attack, moreover after this process it will then release the selected packets of data. Consider an example, where the intruder releases the data packets from one particular initial point, and also it can release the packets probably in the way otherwise it will release the packets in some various specific dedicated routes. Since it is then discussed in $\mathrm{BH}$ as well as $\mathrm{GH}$ attacks with a slightly unusual approach of information packet releasing hits, where the packet forwarding will be simply failed due to unidentified reason such as Sybil Attack [20].

\subsection{Sybil Attack}

In a MANET infrastructure each of the nodes follows with a totally different address to take part in the routing, by which all the different nodes will be brought into the notice. However, it is clear that there will not be any authority dealing the MANETs centrally to control to confirm these identities and due to this attacker exploits this property and then forwards the control set of information, for example RREQ or R-REP, by making use of the dissimilar identities, it is well-known as a sybil attack which can be denoted as "SY" [21].

\subsection{Point Detection Algorithms}

This paper focuses on algorithms and methodologies which are especially proposed in earlier, to safeguard from the IDS hits. The entire intrusion detection mechanisms which are reviewed as per the taxonomy for the protection methodologies of the taxonomy are categorized. From that, they identified the methods as per the number of types of the attacks, and defined this approach as point detection algorithm which may identify a single group of network layer attacks, general intrusion detection systems (IDSs) and range of attack types [15].

\section{PROTECTING AGAINST DATA PACKET DROPPING}

An important investigation has been carried out for the study and protection against release of data packets from attacks. In this, a security proposal against the dropping of information packet attack based on the supportive involvement of nodes is used. This proposal needs each of the node in the network to maintain the behavior of its neighbors; when it detects packet dropping it starts spying and administers to investigate an attack, after detecting a node which is initiating for dropping of packets this scheme uses a function named as trust collector function to gather trust values from their neighbors of the suspicious nodes. If the nodes are in more number then it has a less trust value for the suspicious node, then they start informing all corresponding nodes about this attacker by signaling a global alarm. The authors compared their performance of this scheme with the watchdog algorithm which is proposed in [21] a result in an improvement in terms of low false alarm rates [23]. Earlier few other proposals have been done based on Neighbor Watch System (NWS) have been proposed earlier for identifying of the misbehaving nodes which involves in dropping of the packets [22,24]. Packet forwarding and misbehavior detection lies on the principle of flow conservation [25], where nodes continuously monitors their neighbors, by maintaining a record of such nodes they hear and then identify the behavior of those nodes once in a while. Misbehaving of nodes is detected by comparing the probable proportion of packets which are dropped with a pre-established misbehavior threshold. An adaptive policy-based edition of this algorithm was proposed in [27]. Alterations of the nodes is achieved in 2 (Two) ways. Firstly, by using a method that calculates the number of

nodes with that of misbehavior detection threshold value. Secondly, the adaptability of the protection mechanism policies that accumulates the changing network conditions alongside with the management objectives [28]. A self structured network layer protocol for delivery of the packet safely in MANETs in scan is proposed [35]. In this the nodes can over hear the packets which are received by their neighbor and keeps a duplicate copy of their neighbor's in the routing table. So, when a neighbor receives a packet it searches for the next hop through its map-reading table. Then, tries to consider the packet as dropped if the spying node do not overhear that packet which is being forwarded from the neighboring node [29]. To mitigate the effect of packet dropping in MANETs, another mechanism is proposed that has 2 parts: one is watchdog and another is path rater [30]. Watchdog uses immoral listing to search those points release data information and path rater that manages every nodes path and then starts reducing its rating when this once learns its packet dropping behavior from watchdog. To moderate the consequence of packet dropping, path rater chooses the appropriate pathway which depends upon points rating. 
Table 2 Shows the various Type of attacks, detection technique used, and routing protocols

\begin{tabular}{|c|c|c|c|c|c|c|c|}
\hline $\begin{array}{l}\text { Title of } \\
\text { Paper }\end{array}$ & $\begin{array}{c}\text { Name of } \\
\text { Algorithm }\end{array}$ & Architecture & Type of attack & $\begin{array}{l}\text { Detection } \\
\text { technique }\end{array}$ & $\begin{array}{l}\text { Routing } \\
\text { protocol }\end{array}$ & Source of data & Contribution \\
\hline $\begin{array}{l}\text { Zhang et } \\
\text { al.[20] }\end{array}$ & None & Distributed & Black hole & $\begin{array}{l}\text { Checking } \\
\text { RREP }\end{array}$ & AODV & $\begin{array}{c}\text { Sequence number of } \\
\text { RREP's from intermediate } \\
\text { nodes }\end{array}$ & $\begin{array}{l}\text { Black hole detection } \\
\text { mechanism }\end{array}$ \\
\hline $\begin{array}{l}\text { Sen.et.al } \\
\text { [24] }\end{array}$ & None & Hierarchical & $\begin{array}{l}\text { Grey hole } \\
\text { attack }\end{array}$ & $\begin{array}{l}\text { Monitoring } \\
\text { behavior in } \\
\text { terms of } \\
\text { RREP }\end{array}$ & AODV & $\begin{array}{l}\text { Neighbor data collection } \\
\text { module Transmitted }\end{array}$ & $\begin{array}{l}\text { Grey hole detection for } \\
\text { AODV }\end{array}$ \\
\hline $\begin{array}{l}\text { Yang } \\
\text { et.al.[29] }\end{array}$ & Scan & Distributed & $\begin{array}{l}\text { Data Packet } \\
\text { dropping }\end{array}$ & $\begin{array}{l}\text { Information } \\
\text { cross } \\
\text { validation }\end{array}$ & $\begin{array}{c}\text { Isolate } \\
\text { attackers }\end{array}$ & Collaborative monitoring & $\begin{array}{l}\text { Provide secure packet } \\
\text { delivery in MANET's }\end{array}$ \\
\hline $\begin{array}{l}\text { Zhang \& } \\
\text { Lee [33] }\end{array}$ & None & $\begin{array}{l}\text { Distributed } \\
\text { Peer to peer }\end{array}$ & $\begin{array}{c}\text { Various } \\
\text { network layer }\end{array}$ & ABID & $\begin{array}{l}\text { AODV, } \\
\text { DSR }\end{array}$ & System Events & $\begin{array}{l}\text { Agent based IDS } \\
\text { architecture }\end{array}$ \\
\hline $\begin{array}{c}\text { Yi et. Al } \\
{[34]}\end{array}$ & None & $\begin{array}{l}\text { Hierarchical } \\
\text { Distributed }\end{array}$ & $\begin{array}{l}\text { DOS attacks } \\
\text {,Routing loop }\end{array}$ & Other IDS & DSR & $\begin{array}{l}\text { DSR Routing } \\
\text { Specification }\end{array}$ & FSM to detect attacks \\
\hline $\begin{array}{l}\text { Nadeem } \\
\text { and } \\
\text { Howarth } \\
{[15]}\end{array}$ & AIDP & $\begin{array}{l}\text { Hierarchical, } \\
\text { clustered }\end{array}$ & DoS attacks & ABID & AODV & Routing information & $\begin{array}{l}\text { ABID for detecting DoS } \\
\text { attacks in MANETs }\end{array}$ \\
\hline Smith [35] & Nil & Distributed & Not Specified & KBID & General & Audit Trials & KBID using mobile agents \\
\hline
\end{tabular}

\section{INTRUSION DETECTION SYSTEMS}

IDS are divided into 3 major categories based on the type of methodology used for detection which are employed namely:

Anomaly-based intrusion detection (ABID), which is wellknown as Behavior-based intrusion detection system.

- $\quad$ Misuse detection, is also called as knowledge-based intrusion detection (KBID).

- $\quad$ Specification-based intrusion detection (SBID) [15].

The categorization is done as per the intrusion detection technique used, either Anomaly Based Intrusion Detection, Knowledge Based Intrusion Detection, Signature Based Intrusion Detection, or a hybrid of these, or some other mechanism. Similarly, many of the IDS techniques used are depicted in table 2.

\subsection{Anomaly-Based Intrusion Detection}

The ABID systems flagged as irregular observed behaviors which move away extensively through the usual profile. These ABID methods are well-known as behavior-based intrusion detection (BBID), in which the representation of usual behavior of the network is extracted, and then this model is then differentiated with the existing behavior of the network to identify intrusion in the network. Anomaly detection systems typically comprises of 2 phases namely testing and training [15] [18].

\subsection{Knowledge-Based Intrusion Detection}

KBID system is another technique that stores nodes information in an information base which has patterns or signature of well-versed hits and then starts looking for these styles in a trial to identify misbehaving nodes. In other words, Knowledge Based Intrusion Detection system has much information about precise attacks and tries an attempt to use them. This Knowledge Based Intrusion Detection systems system initiate an alerts in the form of an alarm when an attack is identified [15]. Ultimately, this KBID system relies on knowledge of the previous hits to surveillance new attacks.

\subsection{Other Intrusion Detection Proposals}

In another study a clustered detection approach where sometimes an individual node is selected as the monitoring node for detection of intruders [32]. Similarly, in another survey there are examples of intrusion detection mechanisms which will detect a wide range of attack types as openly stated using the type of ID techniques they had studied and analyzed the probability of mobile agents for MANET intrusion detection and accomplished that many of the mobile agent's features assure the requirements for MANET using IDS. They trust that mobile agents will run executing their programs without being disturbing other nodes by the originating node status which will misguide the nodes with the relevance to the other nodes similar to the challenges faced in MANETs, such as dropping network load, conserving of bandwidth and having strong fault-tolerant behavior [31]. Further, it will start pointing out those mobile agents that has safety vulnerabilities, which might be one of such aspect why they are yet not being used broadly for IDS [15].

\section{CONCLUSION AND FUTURE WORK}

The main characteristic of Mobile Adhoc networks is that, it is infrastructure less and hence has minor control over the centralized behavior and falls prey to various types of attacks. Specifically, an intrusion detection attacks which has become bit difficult task and thus makes MANETS more Vulnerable which can be easily harmed at the Network Layer.

This Surveillance system for overcoming the intruders can be avoided by the use of effective Machine Learning techniques, at the standard Network Layer and also by using appropriate Intrusion Detection techniques that detects and predict the hidden mischievous malfunctioning of those nodes which are altered by intruders which is my future work. 


\section{ACKNOWLEDGMENTS}

I would like to express my sincere thanks to all those without whom the quality of the paper would not be possible including the anonymous reviewers and the work cited as references for this paper.

\section{REFERENCES}

[1] Aumreesh Kumar, Saxena ,Sitesh Sinha, Piyush Shukla, "A Review on Intrusions Detection System in Mobile Ad-Hoc Network" Proceeding of International conference on Recent Innovations is Signal Processing and Embedded Systems (RISE - 2017), 27th - 29th October, IEEE, 2017.

[2] Mohamed Elboukhari, Mostafa Azizi and Abdelmalek Azizi "Intrusion Detection Systems in mobile ad hoc networks: A survey" 5th Workshop on Codes, Cryptography and Communication Systems (WCCCS), Morocco, pp.136-141, November, IEEE, 2014.

[3] Sara Chadli, Mohamed Emharraf, Mohammed Saber and Abdelhak Ziyyat, "Combination of Hierarchical and Cooperative Models of an IDS for MANETs" Tenth International Conference on Signal-Image Technology and Internet-Based Systems, Morocco, pp. 230- 236, November, IEEE, 2014.

[4] Sayan Banerjee, Roshni Nandi, Rohan Dey and Himadri Nath Saha "A review on different Intrusion Detection Systems for MANET and itsvulnerabilities" International Conference and Workshop on Computing and Communication (IEMCON), India, pp. 1-7, October, IEEE, 2015.

[5] T. Poongothai and K. Duraiswamy " Intrusion detection in mobile AdHoc networks using machine learning approach" International Conference on Information Communication and Embedded Systems (ICICES2014), India, pp. 1 - 5, February, IEEE, $2014 .$.

[6] K. Pavani and A. Damodaram, "Intrusion detection using MLP for MANETs" Third International Conference on Computational Intelligence and Information Technology (CIIT 2013), India, pp. 440 - 444, October, IEEE, 2013.

[7] Udaya Sampath K. Perera Miriya Thanthrige, Jagath Samarabandu, Xianbin Wang, "A Machine Learning Techniques for Intrusion Detection on Public Dataset", in proceedings of IEEE Canadian Conference on Electrical and Computer Engineering (CCECE), IEEE, 2016.

[8] J. Canady and J. Harrell, "A comparative analysis of current intrusion detection technologies", in Proceedings of the Fourth Technology for Information Security Conference, vol. 96. Cite seer, 1996.

[9] M. Salour and X. Su, "Dynamic two-layer signaturebased ids with unequal databases", in Information Technology, 2007. ITNG '07.Fourth International Conference on, April pp.77-82, 2007,

[10] N.H. Saeed, M.F. Abbod, H.S. Al-Raweshidy Wireless Network and Communication Centre (WNCC) School of Engineering and Design Brunel University West London, Uxbridge,UK, IMAN: "An Intelligent MANET Routing System"

[11] Erick Peterson and Marco Antonio, "A Novel Online CEP Learning engine for MANET IDS", published in
IEEE 9th Latin-American Conference on Communications (LATINCOM), 2017.

[12] Samreen Banu, Kazi ,Mohammed Azharuddin Adhoni, "Secure IDS to detect Malevolent Node in MANETS", International Conference on Electrical, Electronics and Optimization Techniques (ICEEOT), 2016

[13] .David B. Johnson David A. Maltz Josh Broch, "DSR: "The Dynamic Source Routing Protocol for Multi-Hop Wireless Ad Hoc Networks", ACM, 2001.

[14] Yong guang Zhang, "Intrusion Detection In Wireless Adhoc networks", In proceedings of the 6th annual international conference on Mobile computing and networking, pp. 275-283, ACM, 2000.

[15] Adnan Nadeem, and Michael P. Howarth, "A Survey of MANET Intrusion Detection \& Prevention Approaches for Network Layer Attacks", IEEE Communications Surveys \& Tutorials, Vol. 15, Issue 4, 2013.

[16] IETF Mobile Adhoc Networks Working Group (MANET), IETF Website, www. Ietf.org/dyn/wg/charter/manet-charter.html.

[17] IETF Ad-Hoc Networks Autoconfigurations (autoconf) Working Group, IETF website http://datatracker.ietf.org/wg/autoconf/charte/.

[18] IEEE Std 802.11-2007, IEEE standard for information technology- Telecommunication and information exchange between systems- Local and metropolitan area network-Specific requirement, Part 11 Wireless LAN medium access control and physical layer specifications, June 2007.

[19] S.KurosawaandA.Jamalipour,"DetectingBlackholeAttack onAODV-based Mobile AdHoc Networks by Dynamic Learning method", International Journal of Network Security, Vol.5, No.3, pp 338-345, November 2007.

[20] J.Sen, M.Chandra, S.G. Harihara, H.Reddy P.Balamuralidhar, "A Mechanism for Detection of Gray Hole Attacks in Mobile Ad Hoc Networks", Proc. IEEE International Conference on Information Communication and Signal Processing ICICS, Singapore, Dec.2007.

[21] C.Piro, C.Shields and B.Levine, "Detecting the Sybil Attack in Mobile Ad hoc Networks", Proc. IEEE International Conference on Security and Privacy in Communication Networks, Aug-Sep.2006.

[22] J. Sen, M. Chandra, P. Balamurlidhar, S.G. Harihara and H.Reddy, "A Distributed Protocol for Detection of Packet Dropping Attack in Mobile Ad hoc Networks", Proc. IEEE Conference on Telecommunication and Malaysian International Conference on Communication (ICT-MICC), 2007.

[23] S. Marti, T.J. Giuli, K.Lai and M. Baker, "Mitigating Routing Misbehavior in Mobile Ad Hoc Networks", Proc. International Conference on Mobile Computing and Networking, pp. 255- 265, 2000.

[24] S.B.LeeandY.H.Choi,“ A Resilient Packet Forwarding Scheme against Maliciously Packet Dropping Nodes in Sensor Networks", Proc. ACM workshop on Security of Ad Hoc and Sensor Networks (SANS2006),pp59-70, USA, Oct. 2006.

[25] O.F. Gonzalez-Duque, M. Howarth and G. Pavlou, "Detection of Packet Forwarding Misbehavior in Mobile 
Ad hoc Networks", Proc. International Conference on Wired/Wireless Internet Communications (WWIC 2007), pp 302-314, Portugal, June 2007.

[26] K. Pavani and A. Damodaram "Intrusion detection using MLP for MANETs" Third International Conference on Computational Intelligence and Information Technology (CIIT 2013), India, pp. 440 - 444, October-2013.

[27] Oscar F. Gonzalez, Godwin Ansa, Michael Howarth, and George Pavlou" Detection and Accusation of Packet Forwarding Misbehavior in Mobile Ad-Hoc Networks" Journal of internet engineering, VOL. 2, NO. 1, June2008

[28] Sunilkumar S. Manvia , Lokesh B. Bhajantrib , and Vittalkumar K. Vaggac" Routing Misbehavior Detection in MANETs Using 2ACK"Journal of telecommunications and information technology,2010.

[29] Hao Yang, James Shu, Xiaoqiao Meng, Songwu Lu" SCAN: Self-Organized Network-Layer Security in Mobile Ad Hoc Networks". : IEEE Journal on Selected Areas in Communications (Volume: 24 , Issue: 2 , Feb. 2006 ).

[30] N. Soganile1 , T. Baletlwa2 , and B. Moyo2" Hybrid
Watchdog and Pathrater algorithm for improved security in Mobile Ad Hoc Networks" Int'l Conf. Wireless Networks ICWN'15.

[31] Nitin Goyal, Alka Gaba "A review over MANET- Issues and Challenges" International Journal of Enhanced Research in Management \& Computer Applications, ISSN: 2319-7471 Vol. 2, Issue 4, April-2013, pp: (1628), Available online at: www.erpublications.com.

[32] Saravanan Kumarasamy, Hemalatha B and Hashini P "Cluster Based cost efficient intrusion detection system for MANET” arXiv preprint arXiv:1311.1446,2013.

[33] Y. Zhang and W. Lee, "Intrusion Detection in Wireless Ad-Hoc Networks," Proceedings of the $6^{\text {th }}$ International Conference on Mobile Computing and Networking, MobiCom 2000, pp. 275-283, August 2000.

[34] P. Yi, Y. Jiang, Y. Zhong and S. Zhang, "Distributed Intrusion Detection for Mobile Ad Hoc Networks", Proc. IEEE Application and Internet Workshop, 2005

[35]A.B.Smith,"An examination of intrusion detection architecture for wireless AdHoc networks",Proc. National Colloquium for information system security education, May 2001. 